\title{
Identification of a New Cytotoxic Biflavanone from Selaginella doederleinii
}

\author{
Na-Youn LeE, ${ }^{a}$ Hye-Young Min, ${ }^{a}$ Jun LeE,,${ }^{a, b}$ Joo-Won NAm, ${ }^{a}$ Yoo-Jin Lee, ${ }^{a}$ Ah-Reum Han, ${ }^{a}$ \\ Adam WirYawan, ${ }^{c}$ Wahyu SuPrapto, ${ }^{d}$ Sang Kook LeE, ${ }^{a}$ and Eun-Kyoung Seo ${ }^{*, a}$ \\ ${ }^{a}$ College of Pharmacy and The Center for Cell Signaling \& Drug Discovery Research, Ewha Womans University; Seoul \\ 120-750, Korea: ${ }^{b}$ Department of Herbal Pharmaceutical Development, Korea Institute of Oriental Medicine; Daejeon \\ 305-812, Korea: ' Brawijaya University; Malang 65145, Indonesia: and ${ }^{d}$ Batu Herba Medica Centre; East Java, \\ Indonesia. Received May 22, 2008; accepted June 23, 2008; published online June 26, 2008
}

\begin{abstract}
A new biflavanone, 2,2",3,3"-tetrahydrorobustaflavone 7,4',7"-trimethyl ether (1) was isolated from the whole plant of Selaginella doederleinii HIERON. (Selaginellaceae) together with the known biflavonoid, robustaflavone $7,4^{\prime}, 7^{\prime \prime}$-trimethyl ether (2) as the cytotoxic constituents against the three human cancer cell lines, HCT, NCIH358, and K562. The structure of the new compound 1 was elucidated by spectral analysis including various 1Dand 2D-NMR experiments.
\end{abstract}

Key words Selaginella doederleinii; Selaginellaceae; biflavanone; cytotoxicity

The genus Selaginella is composed of about 700 species and belongs to the family Selaginellaceae. In particular, $\mathrm{Se}$ laginella doederleinii HIERON. has been used as a traditional Chinese medicine ${ }^{1)}$ which is a well-known perennial Pteridophyte plant growing in South and Southwestern China at low altitude. $^{2)}$ It has been used for the treatment of cancer and cardiovascular diseases. ${ }^{1-3)}$ Several biflavonoids, lignans, and alkaloids have been reported from this plant ${ }^{4-7)}$ together with some biological activities such as cytotoxicity, ${ }^{4)}$ antihuman immunodeficiency virus (HIV) activity, inhibition of human DNA polymerase, and anti-inflammatory effects. ${ }^{4-7}$ ) In the present study, a new biflavanoid $\mathbf{1}$ was isolated from the whole plant of $S$. doederleinii with the known biflavonoid, robustaflavone $7,4^{\prime}, 7^{\prime \prime}$-trimethyl ether (2). ${ }^{8)} \mathrm{Com}$ pound 2 was isolated from the family Sellaginellaceae for the first time. They were evaluated for their cytotoxic activity together with the known biflavonoid, robustaflavone 4'-methyl ether (3) which was previously reported from this plant.

Compound 1 was obtained as pale yellow powder and showed a molecular ion peak at $[\mathrm{M}+\mathrm{H}]^{+} \mathrm{m} / \mathrm{z} \quad 585.1755$ (Calcd 585.1753) in the high-resolution ESI mass spectrometry, which was consistent with the molecular formula of $\mathrm{C}_{33} \mathrm{H}_{28} \mathrm{O}_{10}$. The ${ }^{1} \mathrm{H}-\mathrm{NMR}$ spectrum of 1 showed two downfield signals at $\delta_{\mathrm{H}} 12.04$ and 12.10, indicating the presence of hydrogen-bonded hydroxyls. Two sets of ABX signals at $\delta_{\mathrm{H}}$ $2.79(1 \mathrm{H}, \mathrm{dd}, J=17.0,2.6 \mathrm{~Hz}), 3.17(1 \mathrm{H}, \mathrm{dd}, J=17.0$, $13.0 \mathrm{~Hz}), 5.43(1 \mathrm{H}, \mathrm{dd}, J=13.0,2.6 \mathrm{~Hz}) / 2.85(1 \mathrm{H}, \mathrm{dd}$, $J=17.0,2.6 \mathrm{~Hz}), 3.12(1 \mathrm{H}, \mathrm{dd}, J=17.0,2.6 \mathrm{~Hz}), 5.43(1 \mathrm{H}$,
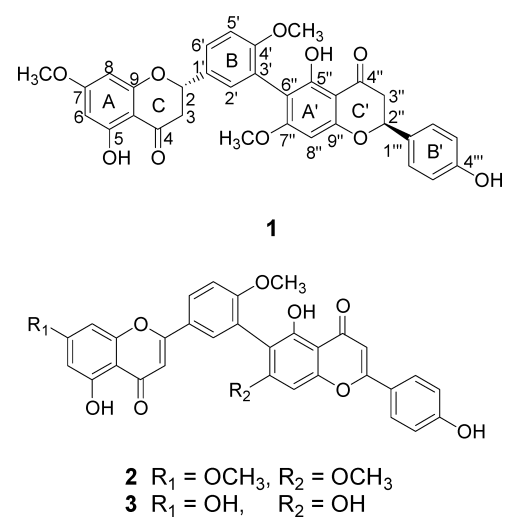

* To whom correspondence should be addressed. e-mail: yuny@ewha.ac.kr dd, $J=13.0,2.6 \mathrm{~Hz}$ ) were corresponding to the protons, $\mathrm{H}-2$, $\mathrm{H}-3 / \mathrm{H}-2^{\prime \prime}, \mathrm{H}-3^{\prime \prime}$ of two flavanone moieties, respectively. Three methoxyl protons at $\delta_{\mathrm{H}} 3.81(3 \mathrm{H}, \mathrm{s}), 3.80(3 \mathrm{H}, \mathrm{s})$, and $3.75(3 \mathrm{H}, \mathrm{s})$ were assigned at $\mathrm{C}-4^{\prime}, \mathrm{C}-7$, and $\mathrm{C}-7^{\prime \prime}$, respectively, according to the three-bond heteronuclear multiple bond connectivity (HMBC) connectivities with the carbon signals at $\delta$ c $158.2,170.0$, and 165.4 , respectively. Two meta-coupled aromatic proton signals at H-6 and H-8 appeared at $\delta_{\mathrm{H}} 6.06$ and $6.05(J=2.6 \mathrm{~Hz})$. The $\mathrm{A}_{2} \mathrm{X}_{2}$ coupling system was established from the signals at $\delta_{\mathrm{H}} 6.90(2 \mathrm{H}, \mathrm{d}$, $\left.J=8.2 \mathrm{~Hz}, \mathrm{H}-3^{\prime \prime \prime}, 5^{\prime \prime \prime}\right)$ and $7.36\left(2 \mathrm{H}, \mathrm{d}, J=8.2 \mathrm{~Hz}, \mathrm{H}-2^{\prime \prime \prime}, 6^{\prime \prime \prime}\right)$. The HMBC correlations of C-6"/OH-5", H-2', H- $8^{\prime \prime}$ and C$3^{\prime} / \mathrm{H}-5^{\prime}, \mathrm{H}-8^{\prime \prime}$ indicated that $\mathbf{1}$ had an interflavonoid linkage between C-3' and C-6" corresponding to its biflavonoid skeleton of robustaflavone series. ${ }^{1,4,8)}$ The ${ }^{1} \mathrm{H}$ - and ${ }^{13} \mathrm{C}$-NMR signals of 1 were assigned unambiguously by further detailed analysis of the ${ }^{1} \mathrm{H}-{ }^{13} \mathrm{C}$ HSQC, ${ }^{1} \mathrm{H}-{ }^{13} \mathrm{C} \mathrm{HMBC}$, and ${ }^{1} \mathrm{H}-{ }^{1} \mathrm{H}$ correlative spectroscopy (COSY) spectra as shown in Fig. 1.

To resolve the absolute configuration at $\mathrm{C}-2$ and $\mathrm{C}-2$ " of $\mathbf{1}$, the circular dichroism (CD) experiment was performed. The

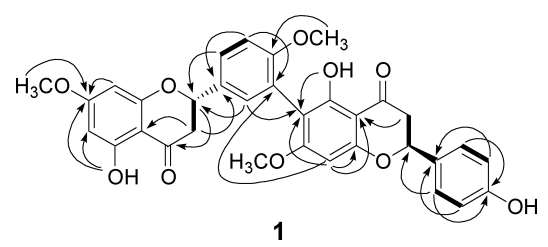

Fig. 1. Selected Correlations Observed in the $\operatorname{HMBC}(\rightarrow)$ and $\operatorname{COSY}(-)$ NMR Spectra of 1

Table 1 . The Cytotoxicity of Isolates from S. doederleinii ${ }^{a)}$

\begin{tabular}{cccc}
\hline \hline \multirow{2}{*}{ Compounds } & \multicolumn{3}{c}{ Cell lines $^{b)}$} \\
\cline { 2 - 4 } & HCT116 & NCI-H358 & K562 \\
\hline $\mathbf{1}$ & 19.1 & 23.5 & 28.8 \\
$\mathbf{2}$ & 15.6 & 20.1 & 22.5 \\
$\mathbf{3}$ & $>100$ & $>100$ & $>100$ \\
Ellipticin $^{c)}$ & 1.0 & 1.6 & 1.2 \\
\hline
\end{tabular}

a) Results are expressed as $\mathrm{EC}_{50}$ values in $\mu \mathrm{M}$. b) Cell lines: $\mathrm{HCT}=$ colorectal carcinoma; NCI-H358 = bronchioalveolar carcinoma (non-small cell lung cancer cells); $\mathrm{K} 562=$ chronic myelogenous leukemia. c) Ellipticine was used as a positive control. 
CD spectrum of 1 exhibited a positive cotton effect at $328 \mathrm{~nm}$ and a negative cotton effect at $290 \mathrm{~nm}$, respectively, indicating the typical " $S$ " configuration at C-2 in flavanone skeleton. ${ }^{9)}$ Therefore, the structure of $\mathbf{1}$ was determined as a new biflavanone, 2,2",3,3"-tetrahydrorobustaflavone 7,4',7"trimethyl ether. Compounds $\mathbf{2}$ and $\mathbf{3}$ were identified as the known biflavonoids, robustaflavone 7,4',7"-trimethyl ether ${ }^{8)}$ and robustaflavone $4^{\prime}$-methyl ether, ${ }^{1,4)}$ respectively.

Compounds $1\left(\mathrm{IC}_{50} 19.1,23.5,28.8 \mu \mathrm{M}\right)$ and $\mathbf{2}\left(\mathrm{IC}_{50} 15.6\right.$, $20.1,22.5 \mu \mathrm{M})$ exhibited cytotoxic activity against the three human cancer cell lines, HCT116, NCI-H358, and K562, whereas compound $\mathbf{3}$ did not show activity. Compounds 1 and 2 have methoxyls at C-7 and C-7" in their flavonoid skeleton, whereas compound $\mathbf{3}$ has hydroxyls at C-7 and C7", indicating the methoxyls at C-7 and C-7" seem to be important for their cytotoxic activity, although further studies are needed.

\section{Experimental}

General Optical rotations were measured with a P-1010 polarimeter (JASCO, Japan) at $25^{\circ} \mathrm{C}$. UV spectra $\left(\lambda_{\text {max }}\right)$ were recorded on a U-3000 spectrophotometer (Hitachi, Japan). IR spectra $\left(v_{\max }\right)$ were determined on a FTS 135 FT-IR spectrometer (Bio-Rad, CA, U.S.A.). Circular dichroism measurements were performed using JASCO J-715 CD/ORD spectropolarimeter. The 1D and 2D NMR experiments were conducted on a UNITY INOVA $400 \mathrm{MHz}$ FT-NMR (varian, CA, U.S.A.), and TMS was used as an internal standard. ESI-MS, HR-ESI-MS and FAB-MS were obtained on a JMS 700 Mastation HR-MS spectrometer (JEOL, Japan). TLC analysis was performed on Kieselgel $60 \mathrm{~F}_{254}$ (Merck, Germany) plates (silica gel, $0.25 \mathrm{~mm}$ layer thickness), with compounds visualized by dipping plates into $10 \%(\mathrm{v} / \mathrm{v}) \mathrm{H}_{2} \mathrm{SO}_{4}$ reagent (Aldrich) followed by charring at $110^{\circ} \mathrm{C}$ for $5-$ 10 min. Silica gel (230 - 400 mesh, Merck, Germany), and RP-18 (YMCGEL ODS-A, $12 \mathrm{~nm}, \mathrm{~S}-150 \mu \mathrm{m}$ ) were used for column chromatography. All solvents used for chromatographic separations were distilled before use.

Plant Material The whole plants of $S$. doedeleinii Hieron. were collected at Batu Medica Centre, East Java, Indonesia, in May 2005 and were identified by Prof. Adam Wiryawan (Brawijaya University, Malang 65145, Indonesia). A voucher specimen has been deposited at the Batu Herba Medica Centre.

Extraction and Isolation The dried and milled plant material $(335 \mathrm{~g})$ was extracted with $\mathrm{MeOH}(3 \times 81)$ by maceration. The $\mathrm{MeOH}$ extracts $(25 \mathrm{~g})$ were separated by liquid column chromatograph [(glass column $(6.4 \times 22 \mathrm{~cm})$; silica gel $(230-400 \mathrm{mesh}, 360 \mathrm{~g})]$ using gradient mixtures of $n$-hexane-EtOAc-MeOH gradients (from $n$-hexane-EtOAc $=49: 1$ to $1: 1$, $n$-hexane-EtOAc- $\mathrm{MeOH}=1: 1: 0$ to $0: 0: 1)$ as mobile phases to give 13 fractions (FI-FXIII). Fraction FXII (7.5 g, $n$-hexane-EtOAc-MeOH= $1: 1: 5)$ was subjected to Sephadex LH-20 column chromatography using $100 \% \mathrm{MeOH}$ as a solvent system (F01-F05). The F01 fraction $(15 \mathrm{mg})$ was chromatographed over reverse-phase column $(\phi 2.2 \mathrm{~cm}$; ODS-A, $12 \mathrm{~nm}$, S$150 \mu \mathrm{m}, 40 \mathrm{~g}$ ) with $\mathrm{MeOH}-\mathrm{H}_{2} \mathrm{O}$ gradient (from $6: 4$ to $8: 2 \mathrm{v} / \mathrm{v}$ ), giving 1 $(2.1 \mathrm{mg}, 0.00039 \% \mathrm{w} / \mathrm{w}) . \mathrm{F} 02(90.1 \mathrm{mg})$ was eluted with $\mathrm{MeOH}-\mathrm{H}_{2} \mathrm{O}$ $(\phi 2.2 \mathrm{~cm}$; ODS-A, $12 \mathrm{~nm}, \mathrm{~S}-150 \mu \mathrm{m}, 40 \mathrm{~g})$ by reverse-phase column chromatography, providing 5 fractions (F0201-F0205). Subfraction, F0205 $(30.5 \mathrm{mg})$ was further separated using a preparative TLC $\left(\mathrm{CHCl}_{3}-\mathrm{MeOH}=\right.$ $49: 1)$ to afford compound 2 ( $3 \mathrm{mg}, 0.00056 \%, R f 0.25)$. F05 fraction $(1.1 \mathrm{~g})$ was further fractionated by flash silica gel column chromatography $\left(\phi 2.8 \mathrm{~cm} ; 230-400\right.$ mesh, $70 \mathrm{~g}$ ) using $\mathrm{CHCl}_{3}-\mathrm{MeOH}$ (gradient from 49:1 to $\mathrm{MeOH} 100 \% \mathrm{v} / \mathrm{v})$ as eluents, affording $3(14.4 \mathrm{mg}, 0.0026 \% \mathrm{w} / \mathrm{w})$.

2,2",3,3"-Tetrahydrorobustaflavone 7,4',7"-Trimethyl Ether (1): A pale yellow powder; $[\alpha]_{\mathrm{D}}^{25}-16.4^{\circ}\left(c=0.17, \mathrm{CHCl}_{3}\right) ; \mathrm{CD}\left(c=2.0 \times 10^{-3} \mathrm{~mm}\right.$, $\left.\mathrm{CHCl}_{3}\right) \Delta \varepsilon_{289}-6.70, \Delta \varepsilon_{327}+2.40 ; \mathrm{IR}(\mathrm{KBr}) \mathrm{cm}^{-1}: 2930,1735,1639,1574$, $1455,1204,1110,1028,816,758 ;{ }^{1} \mathrm{H}-\mathrm{NMR}\left(\mathrm{CDCl}_{3}, 400 \mathrm{MHz}\right) \delta: 12.10$ $\left(\mathrm{OH}-5^{\prime \prime}\right), 12.04(\mathrm{OH}-5), 7.44\left(1 \mathrm{H}, \mathrm{dd}, J=8.6,2.6 \mathrm{~Hz}, \mathrm{H}-6^{\prime}\right), 7.36(2 \mathrm{H}, \mathrm{d}$, $\left.J=8.2 \mathrm{~Hz}, \mathrm{H}-2^{\prime \prime \prime}, 6^{\prime \prime \prime}\right), 7.28\left(1 \mathrm{H}, \mathrm{d}, J=2.6 \mathrm{~Hz}, \mathrm{H}-2^{\prime}\right), 7.04(1 \mathrm{H}, \mathrm{d}, J=8.6 \mathrm{~Hz}$, H-5'), $6.90\left(2 \mathrm{H}, \mathrm{d}, J=8.2 \mathrm{~Hz}, \mathrm{H}-3^{\prime \prime \prime}, 5^{\prime \prime \prime}\right), 6.18\left(1 \mathrm{H}, \mathrm{s}, \mathrm{H}-8^{\prime \prime}\right), 6.06(1 \mathrm{H}, \mathrm{d}$, $J=2.6 \mathrm{~Hz}, \mathrm{H}-6), 6.05(1 \mathrm{H}, \mathrm{d}, J=2.6 \mathrm{~Hz}, \mathrm{H}-8), 5.43(1 \mathrm{H}, \mathrm{dd}, J=13.0,2.6 \mathrm{~Hz}$, $\mathrm{H}-2 \beta), 5.41\left(1 \mathrm{H}, \mathrm{dd}, J=13.0,2.6 \mathrm{~Hz}, \mathrm{H}-2^{\prime \prime} \beta\right), 3.81\left(3 \mathrm{H}, \mathrm{s}, \mathrm{OC}_{3}-4^{\prime}\right), 3.80$ $\left(3 \mathrm{H}, \mathrm{s}, \mathrm{OC}_{3}-7\right), 3.75\left(3 \mathrm{H}, \mathrm{s}, \mathrm{OCH}_{3}-7^{\prime \prime}\right), 3.17(1 \mathrm{H}, \mathrm{dd}, J=17.0,13.0 \mathrm{~Hz}, \mathrm{H}-$ $3 \alpha), 3.12\left(1 \mathrm{H}, \mathrm{dd}, J=17.0,2.6 \mathrm{~Hz}, \mathrm{H}-3^{\prime \prime} \alpha\right), 2.85(1 \mathrm{H}, \mathrm{dd}, J=17.0,2.6 \mathrm{~Hz}, \mathrm{H}-$ $\left.3^{\prime \prime} \beta\right), 2.79(1 \mathrm{H}, \mathrm{dd}, J=17.0,2.6 \mathrm{~Hz}, \mathrm{H}-3 \beta) ;{ }^{13} \mathrm{C}-\mathrm{NMR}\left(\mathrm{CDCl}_{3}, 100 \mathrm{MHz}\right) \delta$ : 196.3 (C-4"), 196.1 (C-4), 170.0 (C-7), 165.4 (C-7"), 164.2 (C-5), 163.1 (C9), $163.0\left(\mathrm{C}-9^{\prime \prime}\right), 160.5\left(\mathrm{C}-5^{\prime \prime}\right), 158.2\left(\mathrm{C}-4^{\prime}\right), 156.3\left(\mathrm{C}-4^{\prime \prime \prime}\right), 130.8\left(\mathrm{C}-2^{\prime}\right)$, $130.6\left(\mathrm{C}-1^{\prime \prime \prime}\right), 130.0\left(\mathrm{C}-1^{\prime}\right), 128.0\left(\mathrm{C}-2^{\prime \prime \prime}, 6^{\prime \prime \prime}\right), 127.2\left(\mathrm{C}-6^{\prime}\right), 121.9\left(\mathrm{C}-3^{\prime}\right)$, $115.8\left(\mathrm{C}-3^{\prime \prime \prime}, 5^{\prime \prime \prime}\right), 111.4\left(\mathrm{C}-5^{\prime}\right), 107.5\left(\mathrm{C}-6^{\prime \prime}\right), 103.2(\mathrm{C}-10), 103.0\left(\mathrm{C}-10^{\prime \prime}\right)$ 95.1 (C-6), 94.3 (C-8), 91.4 (C-8"), 79.3 (C-2"), 79.1 (C-2), $56.1\left(\mathrm{OCH}_{3}-4^{\prime}\right)$, $56.0\left(\mathrm{OCH}_{3}-7\right), 55.7\left(\mathrm{OCH}_{3}-7^{\prime \prime}\right), 43.4(\mathrm{C}-2,3), 43.2\left(\mathrm{C}-2^{\prime \prime}, 3^{\prime \prime}\right)$; HMBC correlation: OH-5/C-5, C-6, C-7, C-10; OH-5"/C-5", C-6", C-10"; H-2 $\beta / \mathrm{C}-4$, C3, C-1', C-6'; H-3/C-4, C-10, C-1'; H-6/C-5, C-7, C $8, \mathrm{C}-10 ; \mathrm{H}-8 / \mathrm{C}-6, \mathrm{C}-8$, $\mathrm{C}-9, \mathrm{C}-10 ; \mathrm{H}-2 / \mathrm{C}-2, \mathrm{C}-4^{\prime}, \mathrm{C}-2^{\prime} ; \mathrm{H}-5^{\prime} / \mathrm{C}-1^{\prime}, \mathrm{C}-4^{\prime}, \mathrm{C}-3^{\prime} ; \mathrm{H}^{\prime} 6^{\prime} / \mathrm{C}-1^{\prime}, \mathrm{C}-6^{\prime}, \mathrm{C}-$ $4^{\prime}, \mathrm{C}-6^{\prime \prime} ; \mathrm{H}-2^{\prime \prime} / \mathrm{C}-4^{\prime \prime} ; \mathrm{H}-8^{\prime \prime} / \mathrm{C}-6^{\prime \prime}, \mathrm{C}-7^{\prime \prime}, \mathrm{C}-9^{\prime \prime}, \mathrm{C}-10^{\prime \prime} ; \mathrm{H}-2^{\prime \prime \prime}$ and $6^{\prime \prime \prime} / \mathrm{C}-3^{\prime \prime \prime}, \mathrm{C}-4^{\prime \prime \prime}$, $\mathrm{C}-5^{\prime \prime \prime} ; \mathrm{H}-3^{\prime \prime \prime}$ and $5^{\prime \prime \prime} / \mathrm{C}-1^{\prime \prime \prime}$, C-4"'; $\mathrm{OC}_{3}-7 / \mathrm{C}-7$; $\mathrm{OCH}_{3}-4^{\prime} / \mathrm{C}-4^{\prime} ; \mathrm{OCH}_{3}-7^{\prime \prime} / \mathrm{C}-7^{\prime \prime}$; NOESY correlation: $\mathrm{OCH}_{3}-7 / \mathrm{H}-6$; $\mathrm{OCH}_{3}-4^{\prime} / \mathrm{H}-5^{\prime} ; \mathrm{OCH}_{3}-7^{\prime \prime} / \mathrm{H}-8^{\prime \prime}$; ESI-MS $m / z\left(\%\right.$ rel. int.) $585[\mathrm{M}+\mathrm{H}]^{+}(100), 580$ (44), $546(90), 544(65), 522(40)$, 507 (83), 503 (90); HR-ESI-MS $m / z 585.1753[\mathrm{M}+\mathrm{H}]^{+}$(Calcd for $\left.\mathrm{C}_{33} \mathrm{H}_{28} \mathrm{O}_{10}: 585.1755\right)$.

Robustaflavone 7,4, $7^{\prime \prime}$-Trimethyl Ether (2): Yellow powder; UV (MeOH) $\lambda_{\max }(\log \varepsilon) 341.0 \mathrm{~nm}$ (4.34), $269.0 \mathrm{~nm}$ (4.28); IR (KBr) cm $\mathrm{cm}^{-1} 2894,1649$, 1503, 1349, 1117, 1037, 804; ESI-MS m/z (\%) $581[\mathrm{M}+\mathrm{H}]^{+}$(70), $575(60)$, 573 (55); ${ }^{1} \mathrm{H}-\mathrm{NMR}$ data was in agreement with reported values. $\left.{ }^{8}\right)$

Robustaflavone 4'-Methyl Ether (3): Yellow powder; UV (MeOH) $\lambda_{\max }$ ( $\log \varepsilon$ ) $340.0 \mathrm{~nm}$ (4.38), $270.0 \mathrm{~nm}$ (4.47); IR (KBr) cm $\mathrm{cm}^{-1} 2873,1649,1557$, $1539,1359,1168,1022$; FAB-MS $m / z$ (\% rel. int.) $575[\mathrm{M}+\mathrm{Na}]^{+}(2), 553$ (3), 530 (1), 521 (1), 491 (1); ${ }^{1} \mathrm{H}$ - and ${ }^{13} \mathrm{C}-\mathrm{NMR}$ data were in agreement with reported values. ${ }^{1,4)}$

In Vitro Cytotoxicity Assay Cytotoxic potential was determined as described previously. ${ }^{11)}$ Briefly, cells (in log growth phase) were counted, diluted to $5 \times 10^{4} \mathrm{cells} / \mathrm{ml}$ with fresh medium, and added to 96 -well microtiter plates $(190 \mu \mathrm{l} /$ well $)$ containing test materials $(10 \mu \mathrm{l}$ in $10 \%$ aqueous DMSO). Test plates were incubated for $3 \mathrm{~d}$ at $37^{\circ} \mathrm{C}$ in $\mathrm{CO}_{2}$ incubator. All treatments were performed in triplicate. After the incubation periods, cells were fixed by the addition of $50 \mu \mathrm{m}$ of cold $50 \%$ aqueous trichloroacetic acid $\left(4{ }^{\circ} \mathrm{C}\right.$ for $\left.30 \mathrm{~min}\right)$, washed $4-5$ times with tap water, and air-dried. The fixed cells were stained with sulforhodamine B (SRB) $(0.4 \% \mathrm{w} / \mathrm{w}$ SRB in $1 \%$ aqueous acetic acid) for $30 \mathrm{~min}$. Free SRB solution was then air-dried, the bound dye was solubilized with $200 \mu \mathrm{l}$ of $10 \mathrm{~mm}$ tris-base (pH 10.0), and absorbance was determined at $515 \mathrm{~nm}$ using an ELISA plate reader. Finally, the absorbance values obtained with each of the treatment procedures were averaged, and the averaged value obtained with the zero day control was subtracted. These results were expressed as a percentage, relative to solventtreated control incubations, and $\mathrm{EC}_{50}$ values were calculated using non-linear regression analyses (percent survival versus concentration). Ellipticine was used as a positive control.

Acknowledgments This work was supported by a grant from the Brain Korea 21 Project and by grant No. R15-2006-020 from the National Core Research Center (NCRC) program of the Ministry of Education, Science and Technology (MEST) and the Korea Science and Engineering Foundation (KOSEF) through the Center for Cell Signalling \& Drug Discovery Research at Ewha Womans University.

\section{References}

1) Lu Y., Chen Y., Wen J., Yunnan Zhiwu Yanjiu, 26, 226-228 (2004).

2) Lin R. C., Skaltsounis A.-L., Seguin E., Tillequin F., Koch M., Planta Med., 60, 168-170 (1994).

3) Chao L. R., Seguin E., Tillequin F., J. Nat. Prod., 50, 422-426 (1987).

4) Lin L.-C., Kuo Y.-C, Chou C.-J., J. Nat. Prod., 63, 627-630 (2000).

5) Ma S.-C., But P. P.-H., Ooi V. E.-C., Biol. Pharm. Bull., 24, 311-312 (2001).

6) Liu R. C., Peyroux J., Seguin E., Koch M., Phytother. Res., 5, 188190 (1991).

7) Pan K. Y., Lin J. L., Chen J. S., J. Toxicol. Clin. Toxicol., 39, 637-639 (2001).

8) Gu Y., Xu Y., Fang S., He Q., Zhiwu Xuebao, 32, 631-636 (1990).

9) Gaffield W., Tetrahedron, 26, 4093-4108 (1970).

10) Lee S. K., Cui B., Mehta R. R., Kinghorn A. D., Pezzuto J. M., Chem. Biol. Interact., 115, 215-228 (1998). 